\title{
Author's reply regarding the letter to the editor about our article: A nomogram for predicting occult lymph node metastasis in early hypopharyngeal cancer with cNO
}

\author{
Liang Zhou' ${ }^{1}$ D \\ Received: 5 July 2021 / Accepted: 9 July 2021 / Published online: 15 July 2021 \\ ○ The Author(s), under exclusive licence to Springer-Verlag GmbH Germany, part of Springer Nature 2021
}

\section{Dear Editor,}

We thank for the thoughtful and constructive comment on our recent report: A nomogram for predicting occult lymph node metastasis in early hypopharyngeal cancer with clinically negative cervical lymph node (cN0). We really appreciate the suggestion on limited sample size for the prediction model.

We have admitted that this retrospective single-center study needed a larger sample size and further external validation with more different cohorts as the current nomogram was based on a small cohort.

According to inclusion and exclusion criteria, regrettably, we collected only 78 cases for the study. The sample size was small. Hypopharynx cancer is relatively rare and accounts for roughly $3 \%$ of all head and neck cancers [1]. Patients with early stage of hypopharyngeal squamous cell carcinoma (HPSCC) are frequently asymptomatic and nonspecific. When more evident symptoms such as dysphagia, hoarseness, or sore throat present, most patients are often already in advanced stage, delaying the diagnosis of hypopharyngeal carcinoma. Patients with stage III and IV accounted for 17-27\% and 57-68.5\%, respectively, according to previous studies [2,3]. Moreover, due to rich lymphatic network near the hypopharynx, cervical lymph node metastasis often accompanies HPSCC. $60-80 \%$ of patients presented with cervical lymph node metastases at the time of diagnosis [4]. In addition, a considerable proportion of

This reply refers to the comment available online at https://doi.org/ 10.1007/s00405-021-06782-w.

Liang Zhou

zhoulent@126.com

1 Department of Otolaryngology-Head and Neck Surgery, Eye, Ear, Nose and Throat Hospital, Fudan University, 83 Fenyang Rd, Shanghai 200031, People's Republic of China early hypopharyngeal cancers choose larynx-preserving treatment. The increasing use of non-surgical treatment (radiotherapy and chemotherapy) for hypopharyngeal cancer has apparently led to a decline in rates of laryngopharyngectomy. The above reasons result in low numbers of early hypopharyngeal cancers with cNO. In fact, the number of HPSCC treated in our center is in the domestic leading level.

We are trying to enlarge the clinical sample size. A project to establish a China's special database on hypopharyngeal cancer has been launched. We hope a larger sample size and a multi-center and prospective study to further confirm the outcomes.

\section{References}

1. Garneau JC, Bakst RL, Miles BA (2018) Hypopharyngeal cancer: a state of the art review. Oral Oncol 86:244-250

2. Sewnaik A et al (2005) Treatment of hypopharyngeal carcinoma: analysis of nationwide study in the Netherlands over a 10 -year period. Clin Otolaryngol 30(1):52-57

3. Hall SF et al (2008) The natural history of patients with squamous cell carcinoma of the hypopharynx. Laryngoscope 118(8):1362-1371

4. Buckley JG, MacLennan K (2000) Cervical node metastases in laryngeal and hypopharyngeal cancer: a prospective analysis of prevalence and distribution. Head Neck 22(4):380-385

Publisher's Note Springer Nature remains neutral with regard to jurisdictional claims in published maps and institutional affiliations. 